\title{
Effects of Postoperative Radiotherapy on Leptomeningeal Carcinomatosis or Dural Metastasis after Resection of Brain Metastases in Breast Cancer Patients
}

\section{Boram Ha, MD ${ }^{1}$ \\ Seung Yeun Chung, MD² \\ Yeon-Joo Kim, MD'1 \\ Ho-Shin Gwak, MD \\ Jong Hee Chang, $\mathrm{MD}^{4}$ \\ Sang Hyun Lee, MD \\ In Hae Park, MD \\ Keun Seok Lee, MD ${ }^{6}$ \\ Seeyoun Lee, $\mathrm{MD}^{6}$ \\ Tae Hyun Kim, MD ${ }^{1}$ \\ Dae Yong Kim, MD' \\ Seok-Gu Kang, MD ${ }^{4}$ \\ Chang-Ok Suh, MD²}

*A list author's affiliations appears at the end of the paper.
Correspondence: Yeon-Joo Kim, MD Proton Therapy Center, Research Institute and Hospital, National Cancer Center, 323 Ilsan-ro, Ilsandong-gu, Goyang 10408, Korea

Tel: 82-31-920-1785

Fax: 82-31-920-0149

E-mail: jane2000md@gmail.com

Co-correspondence: Chang-Ok Suh, MD Department of Radiation Oncology,

Yonsei Cancer Center, Yonsei University Health System, 50-1 Yonsei-ro, Seodaemun-gu, Seoul 03722, Korea

Tel: $82-2-2228-8117$

Fax: 82-2-2227-7823

E-mail: cosuh317@yuhs.ac

Received July 12, 2016

Accepted October 15, 2016

Published Online October 31, 2016

*Boram Ha and Seung Yeun Chung contributed equally to this work.

\section{Purpose}

In this retrospective study, we compared the incidence of leptomeningeal carcinomatosis or dural metastasis (LMCDM) in patients who received whole brain radiotherapy (WBRT), partial radiotherapy (PRT), or no radiotherapy (RT) following resection of brain metastases from breast cancer.

\section{Materials and Methods}

Fifty-one patients with breast cancer underwent surgical resection for newly diagnosed brain metastases in two institutions between March 2001 and March 2015. Among these, 34 received postoperative WBRT $(n=24)$ or PRT $(n=10)$ and 17 did not.

\section{Results}

With a median follow-up of 12.4 months (range, 2.3 to 83.6 months), 22/51 patients developed LMCDM at a median of 8.6 months (range, 4.8 to 51.2 months) after surgery. The 18-months LMCDM-free survival (LMCDM-FS) rates were $77.5 \%, 30.0 \%$, and $13.6 \%$, in the WBRT, PRT, and no RT groups, respectively $(p=0.013)$. The presence of a tumor adjacent to cerebrospinal fluid flow and no systemic treatment after treatment for brain metastases were also associated with poor LMCDM-FS rate. Multivariate analysis showed that WBRT compared to PRT ( $p=0.009)$ and systemic treatment $(p<0.001)$ were independently associated with reduced incidence of LMCDM.

\section{Conclusion}

WBRT improved LMCDM-FS rate after resection of brain metastases compared to PRT in breast cancer patients.

\section{Key words}

Breast neoplasms, Meningeal carcinomatosis, Whole brain radiotherapy, Partial radiotherapy 


\section{Introduction}

There has been steady improvement in the survival of breast cancer patients in recent decades; however, late development of brain metastases is an increasing problem in longterm survivors. In fact, breast cancer is the second most common origin of brain metastasis in cancer patients [1]. Intensive treatments, including surgery, radiosurgery, and conventional radiotherapy (RT), can substantially increase survival in some subgroups of patients with a limited number of brain metastases, controlled extracranial disease, and good performance status [2,3].

Whole-brain RT (WBRT) following surgical resection of brain metastases has a well-established role in preventing intracranial failure $[4,5]$. Because of concern about possible long-term neurocognitive sequelae, partial radiotherapy (PRT) to the tumor bed has been proposed as a substitute for WBRT [6,7]. However, PRT after surgery is associated with increased risk of leptomeningeal carcinomatosis (LMC) or distant brain failure relative to WBRT [8,9].

The risk of LMC should be carefully considered when applying PRT after surgery because of the theoretical possibility of cerebrospinal fluid (CSF) contamination with tumor cells during surgical resection of brain metastases. A few retrospective studies have compared postoperative WBRT and PRT $[8,9]$. However, these studies included patients with any type of primary cancer, and none of these studies included breast cancer patients alone. Therefore, the present study was conducted to determine the incidence of LMC according to the extent of brain RT after surgical resection of brain metastases in breast cancer patients.

\section{Materials and Methods}

\section{Patients}

We retrospectively reviewed the medical records of breast cancer patients with brain metastases who underwent surgical resection between March 2001 and March 2015 at the National Cancer Center of Korea (Goyang) or between November 2003 and March 2015 at the Severance Hospital of Korea (Seoul). After excluding patients who received RT before surgical resection of brain metastases, 51 patients were included in this study. Overall, 34 patients received postoperative RT, being WBRT in 24 patients and PRT to the tumor bed in 10 patients.

\section{Radiotherapy}

The postoperative treatment strategy; namely, whether or not to give RT and to what extent, was selected according to the physician's preference. A WBRT of 25 or $30 \mathrm{~Gy}$ in 10 fractions (fx) or 30 Gy in $12 \mathrm{fx}$ (median $30 \mathrm{~Gy} / 10 \mathrm{fx}$ ) was administered using a linear accelerator with conventional two opposing lateral fields. There was a difference in treatment strategy in terms of the boost to the tumor bed between the centers. A boost (dose range, 7.5 to $25 \mathrm{~Gy}$; median, $15 \mathrm{~Gy}$ in 3-10 fx) after WBRT was routinely prescribed in one center, but not the other. PRT was applied to the resection cavity with a suitable margin using stereotactic or three-dimensional conformal techniques at various dose fractions: 36 Gy / $6 \mathrm{fx}(\mathrm{n}=4), 45 \mathrm{~Gy} / 15 \mathrm{fx}(\mathrm{n}=2), 45 \mathrm{~Gy} / 10 \mathrm{fx}(\mathrm{n}=1)$, and 36 $\mathrm{Gy} / 12 \mathrm{fx}(\mathrm{n}=1)$. In two patients who were treated with gamma knife radiosurgery, 16 Gy and 7.5 Gy were prescribed to the $50 \%$ isodose line in a single fraction, respectively.

\section{Clinical factors}

In addition to the extent of brain RT, the following clinical variables were reviewed as potential prognostic factors for the recurrence of leptomeningeal disease: age at diagnosis of brain metastasis, systemic disease status (none/stable vs. progressive), biological subtype of primary tumor, Eastern Cooperative Oncology Group (ECOG) performance status, breast specific graded prognostic assessment score (BreastGPA) [10], size and number of metastases, extent of resection (gross total resection vs. subtotal resection), hormone therapy, and systemic treatment. Systemic treatment included chemotherapy, targeted therapy, or both and was given after brain surgery and before the development of LMC or dural metastasis (DM) (LMCDM). Systemic treatment at any time after brain surgery was separately analyzed as a prognostic factor for overall survival (OS). Biological subtypes of primary tumors were classified as follows: estrogen receptorpositive and/or progesterone receptor-positive/human epidermal growth factor receptor (HER2)-negative, HER2positive, and triple-negative according to the previous studies [11]. Continuous variables were dichotomized by their median values. We also analyzed the location of the tumor relative to CSF flow. The tumor was classified as adjacent to CSF flow if there was contact of the tumor's surface with the pia mater or ventricle wall [12].

\section{Outcome evaluation}

The patients were followed-up with magnetic resonance imaging (MRI) at intervals of 3 months after surgery. The primary outcome was the development of LMCDM after treat- 

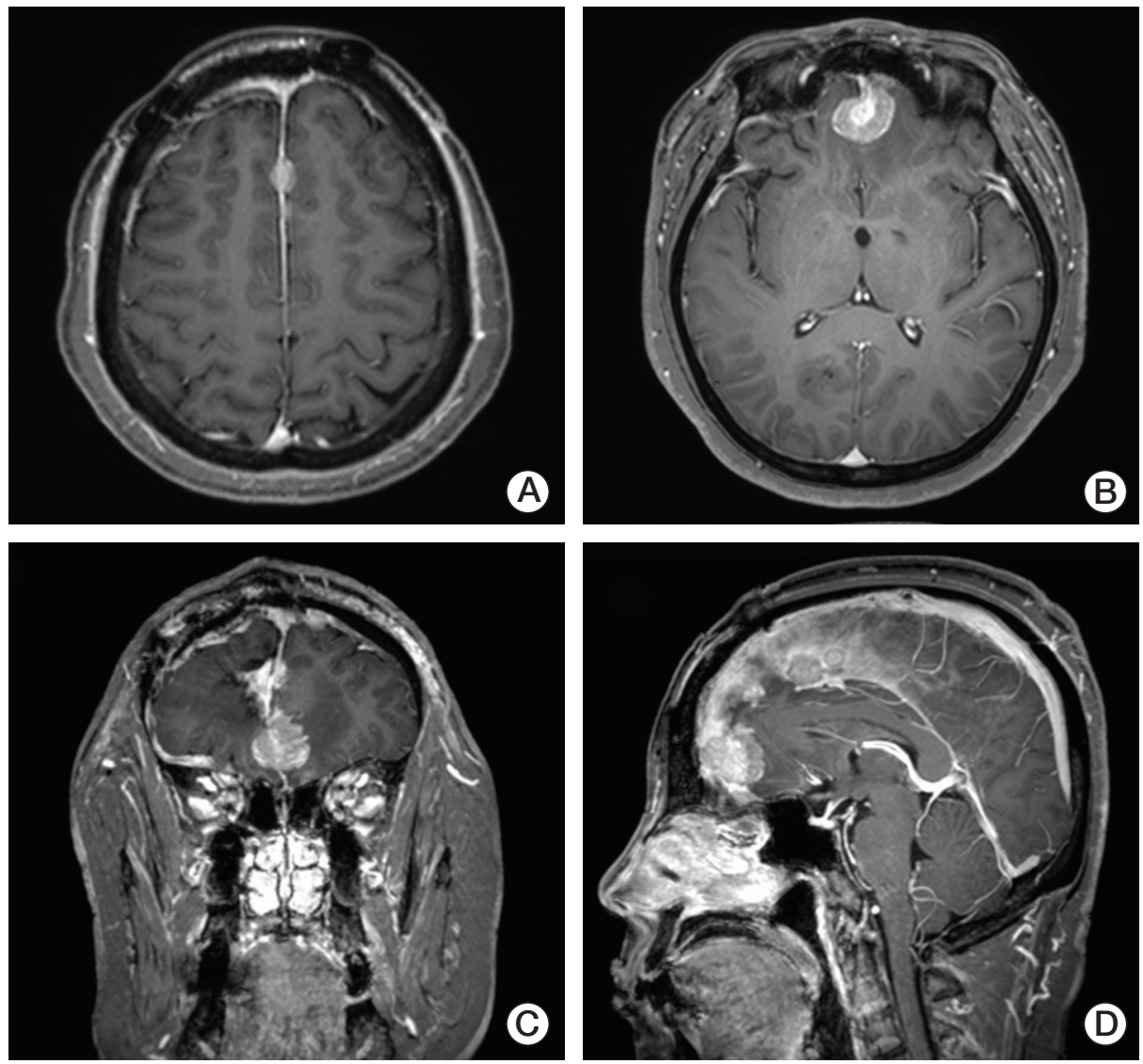

Fig. 1. (A-D) Gadolinum enhanced T1 weighted magnetic resonance imaging (MRI) image of a patient who developed multiple enhancing dural nodules 6 months after surgical resection for brain metastasis and postoperative partial radiotherapy to the tumor bed. Dural metastasis was defined as a presence of such multiple enhancing nodules on the dura mater on MRI.

ment for brain metastases. LMC was diagnosed based on MRI and/or CSF cytology confirmation. The conservative definition of LMC (i.e., presence of malignant cells in CSF or typical leptomeningeal enhancement on MRI in the brain, spinal cord, or cauda equina) was used. DM was defined as a presence of multiple enhancing nodules on the dura mater on MRI (Fig. 1). Both incidence of LMC and DM were analyzed together as a LMCDM.

Local recurrence (LR) was defined as the appearance of new enhancing lesions within the resection cavity. Distant brain recurrence (DBR) was defined as the presence of new metastatic nodules in distinct brain parenchyma outside the resection cavity.

Considering the emerging evidence of the importance of biological subtype and targeted therapy in breast cancer prognosis and treatment [11], subgroup analysis according to the HER2 status was conducted to evaluate the impact of targeted therapy on the development of LMCDM and OS.

\section{Statistical analysis}

Patient characteristics were compared among the three treatment groups using Fisher exact test for categorical variables and the Mann-Whitney U test or one-way analysis of variance for continuous variables, as appropriate. The Kaplan-Meier method was used to assess event-time distributions. The time to recurrence was calculated from the date of surgical resection to the date of MRI showing LR, DBR, or LMCDM. Otherwise, patients were censored at the time of their last MRI or the clinical visit when their neurologic signs were last evaluated. OS was calculated from the date of surgery to the date of death, or living patients were censored at the date of their last clinical visit. Log-rank tests were used to compare the event-time distributions among treatment groups. The Cox proportional hazard model was used in multivariate analysis to identify factors associated with LMCDM by calculating hazard ratio (HR) with $95 \%$ confi- 
Table 1. Patient, tumor, and treatment characteristics

\begin{tabular}{|c|c|c|c|c|c|}
\hline Characteristic & $\underset{(n=51)}{\text { All }}$ & $\begin{array}{l}\text { WBRT } \\
(\mathrm{n}=24)\end{array}$ & $\begin{array}{l}\text { PRT } \\
(\mathrm{n}=10)\end{array}$ & $\begin{array}{l}\text { No RT } \\
(\mathrm{n}=17)\end{array}$ & p-value \\
\hline Age, median (range, yr) & $48(34-75)$ & $46(34-68)$ & $45(34-68)$ & $60(34-75)$ & $0.805^{\mathrm{a})}$ \\
\hline $\begin{array}{l}\text { Duration between brain metastasis and } \\
\text { breast cancer diagnosis, median (range, mo) }\end{array}$ & $35.1(5.0-148.0)$ & $29.9(7.8-148.0)$ & $32.4(9.8-67.5)$ & $41(5.0-115.9)$ & $0.835^{\mathrm{a})}$ \\
\hline \multicolumn{6}{|l|}{ Extracranial disease status } \\
\hline None or stable & $35(69)$ & $15(63)$ & $10(100)$ & $10(59)$ & $0.056^{\mathrm{b})}$ \\
\hline Progressive & $16(31)$ & $9(38)$ & 0 & $7(41)$ & \\
\hline \multicolumn{6}{|l|}{ Biological subtype of primary tumor } \\
\hline ER+ and/or PR+, HER2- & $7(14)$ & $3(13)$ & 0 & $4(24)$ & $0.556^{\mathrm{b})}$ \\
\hline HER2-positive & $31(60)$ & $14(58)$ & $7(70)$ & $10(59)$ & \\
\hline Triple-negative & $13(26)$ & $7(29)$ & $3(30)$ & $3(18)$ & \\
\hline \multicolumn{6}{|l|}{ ECOG performance status } \\
\hline $0-1$ & $47(92)$ & $22(92)$ & $9(90)$ & $16(94)$ & $>0.990^{\mathrm{b})}$ \\
\hline 2 & $4(8)$ & $2(8)$ & $1(10)$ & $1(6)$ & \\
\hline \multicolumn{6}{|l|}{ Breast-GPA } \\
\hline $3.5-4.0$ & $13(26)$ & $5(21)$ & $5(50)$ & $3(18)$ & $0.314^{\mathrm{b})}$ \\
\hline $2.5-3.0$ & $21(41)$ & $9(38)$ & $2(20)$ & $10(56)$ & \\
\hline $1.5-2.0$ & $15(29)$ & $8(33)$ & $3(30)$ & $4(24)$ & \\
\hline $0.5-1.0$ & $2(4)$ & $2(8)$ & 0 & 0 & \\
\hline \multicolumn{6}{|l|}{ No. of brain metastases } \\
\hline 1 & $36(71)$ & $14(58)$ & $7(70)$ & $15(88)$ & $0.117^{\mathrm{b})}$ \\
\hline $2-3$ & $15(29)$ & $10(42)$ & $3(30)$ & $2(12)$ & \\
\hline Size, median (range, $\mathrm{cm}$ ) & $3.5(1.0-8.0)$ & $3.6(1.6-6.0)$ & $3.5(2.0-6.0)$ & $2.9(1.0-8.0)$ & $0.518^{\mathrm{a})}$ \\
\hline \multicolumn{6}{|l|}{ Adjacent to CSF flow ${ }^{c}$} \\
\hline No & $10(20)$ & $4(17)$ & $1(10)$ & $5(29)$ & $0.479^{\mathrm{b})}$ \\
\hline Yes & $40(80)$ & $19(83)$ & $9(90)$ & $12(71)$ & \\
\hline \multicolumn{6}{|l|}{ Extent of resection } \\
\hline GTR & $40(78)$ & $18(75)$ & $7(70)$ & $15(88)$ & $0.447^{\mathrm{b})}$ \\
\hline STR & $11(22)$ & $6(25)$ & $3(30)$ & $2(12)$ & \\
\hline \multicolumn{6}{|l|}{ HRT after brain surgery } \\
\hline Yes & $6(12)$ & $3(6)$ & $2(20)$ & $1(6)$ & $0.549^{b)}$ \\
\hline No & $45(88)$ & $21(88)$ & $8(80)$ & $16(94)$ & \\
\hline \multicolumn{6}{|l|}{$\begin{array}{l}\text { Systemic treatment after brain surgery and } \\
\text { before the development of LMCDM }\end{array}$} \\
\hline No & $21(42)$ & $7(29)$ & $4(40)$ & $10(59)$ & $0.265^{b)}$ \\
\hline Targeted $\mathrm{Tx} \pm \mathrm{CTx}$ & $13(26)$ & $6(25)$ & $4(40)$ & $3(18)$ & \\
\hline CTx & $17(33)$ & $11(46)$ & $2(20)$ & $4(24)$ & \\
\hline \multicolumn{6}{|l|}{$\begin{array}{l}\text { Systemic treatment after brain surgery } \\
\text { (at any time) }\end{array}$} \\
\hline No & $16(31)$ & $7(29)$ & $1(10)$ & $8(47)$ & $0.291^{\mathrm{b})}$ \\
\hline CTx with targeted Tx & $15(29)$ & $6(25)$ & $5(50)$ & $4(24)$ & \\
\hline CTx without targeted Tx & $20(39)$ & $11(46)$ & $4(40)$ & $5(29)$ & \\
\hline
\end{tabular}

Values are presented as number (\%). WBRT, whole brain radiotherapy; PRT, partial radiotherapy; RT, radiotherapy; ER, estrogen receptor; PR, progesterone receptor; HER2, human epidermal growth factor receptor 2; ECOG, Eastern Cooperative Oncology Group; Breast-GPA, breast specific graded prognostic assessment score; CSF, cerebrospinal fluid; GTR, gross total

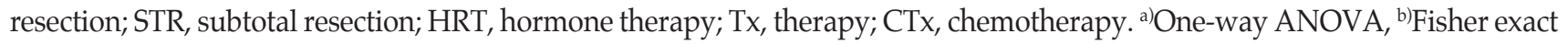
test, ${ }^{c} \mathrm{n}=50$ (WBRT, 23; PRT, 10; no RT, 17). 
dence intervals (CI). A stepwise backward linear regression was employed, and variables with p-values greater than 0.10 were removed from the model. The study was sufficiently powered $(88 \%)$ with a one-sided type I error probability of $5 \%$ to detect a HR of 3.3 for 27 patients who did not received postoperative WBRT relative to 24 patients who did. The HR of 3.3 was based on previous studies in which the HRs for LMC were 2.44 and 5.67 in patients who underwent postoperative PRT compared with those who underwent WBRT $[8,9]$. The review and analysis of patient data in this study were approved by the Institutional Review Boards of both institutions.

\section{Results}

\section{Patient, tumor, and treatment characteristics}

The patient, tumor, and treatment characteristics are summarized in Table 1. The median age was 48 years (range, 34 to 75 years) in all patients, and was higher in the no RT group (60 years) than the WBRT (46 years) and PRT (45 years) groups, although this difference was not statistically significant ( $\mathrm{p}=0.805$ ). A boost RT was applied to the tumor bed in $16 / 24$ patients who received WBRT. Overall, $69 \%$ of patients had no or stable extracranial disease and $71 \%$ had one brain lesion. All patients in the PRT group had no or stable extracranial disease, but the prevalence of extracranial disease was not significantly different among the three groups. The median size of the metastatic brain lesion was $3.5 \mathrm{~cm}$ (range, 1.0 to $8.0 \mathrm{~cm}$ ). Additionally, $80 \%$ of all patients had tumors adjacent to the CSF flow. Hormone therapy was applied in $6 / 19$ patients $(32 \%)$ with hormone receptor-positive primary tumors.

Systemic treatment was administered after surgical resection and before the development of LMCDM in $71 \%$ of patients in the WBRT group, compared with $60 \%$ and $41 \%$ of patients in the PRT and no RT group ( $\mathrm{p}=0.265)$. In a subgroup of 31 patients with HER2-positive primary tumors, 12 patients were given targeted therapy. The regimens of systemic treatment after brain surgery and before the development of LMCDM are listed in S1 Table. The three most commonly used regimens were capecitabine/lapatinib $(37 \%)$, gemcitabine/ cisplatin (33\%), and capecitabine (30\%).

\section{Intracranial recurrence rate and pattern of failure}

During the follow-up period, no intracranial recurrence was noted in 12 of the 24 patients in the WBRT group, two out of 10 in the PRT group, and seven out of 17 in the no RT group (Fig. 2). LR and LMCDM were the major patterns of failure rather than DBR. Eight out of 17 patients $(47 \%)$ in the no RT group developed LR, as did two out of 10 patients $(20 \%)$ in the PRT group and five out of 24 patients (21\%) in

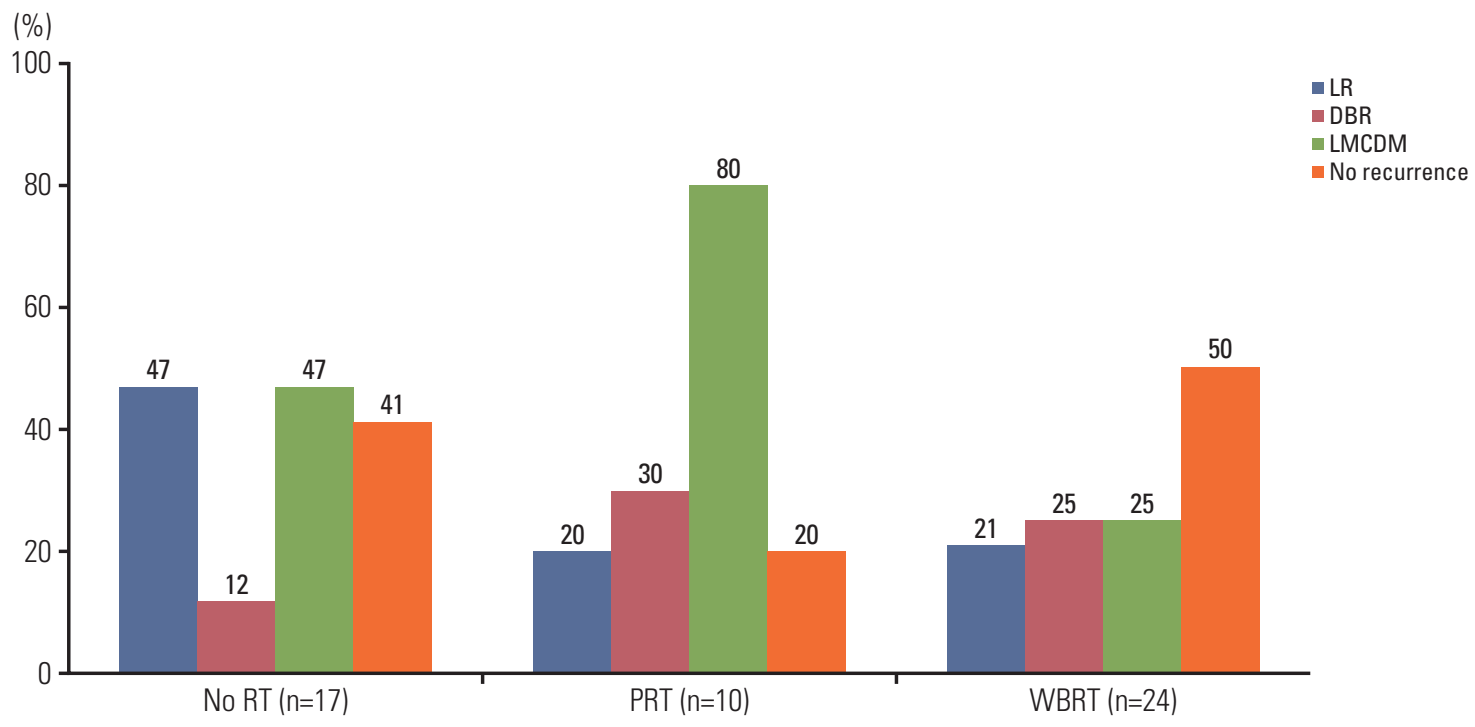

Fig. 2. Intracranial recurrence rate and pattern of failures in three treatment groups: no radiotherapy (RT) group ( $\mathrm{n}=17)$, partial RT (PRT) group $(\mathrm{n}=10)$, and whole brain radiotherapy (WBRT) group $(\mathrm{n}=24)$. $\mathrm{LR}$, local recurrence; $\mathrm{DBR}$, distant brain recurrence; LMCDM, leptomeningeal carcinomatosis or dural metastasis. 
A

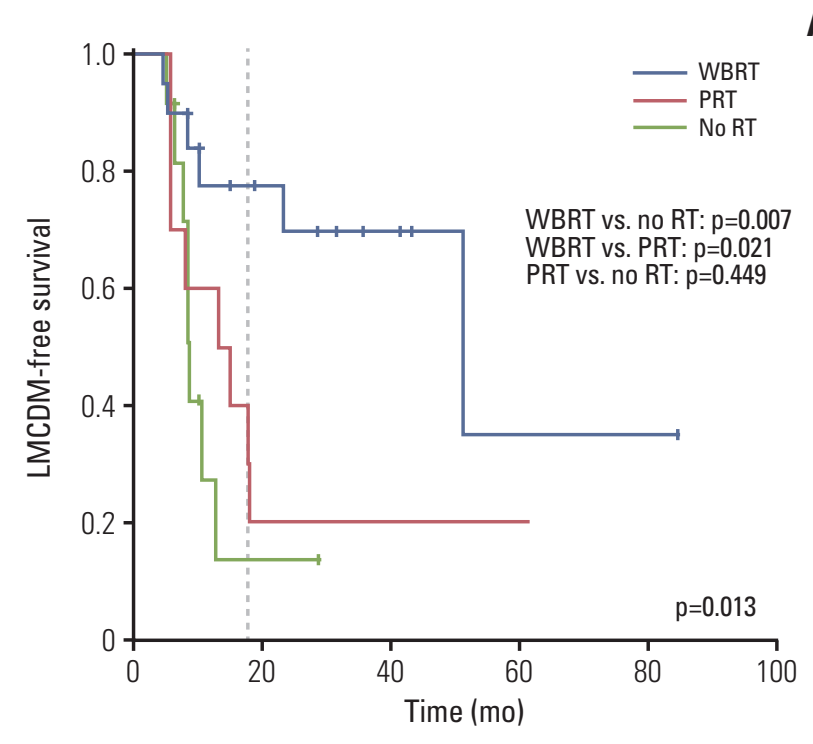

A

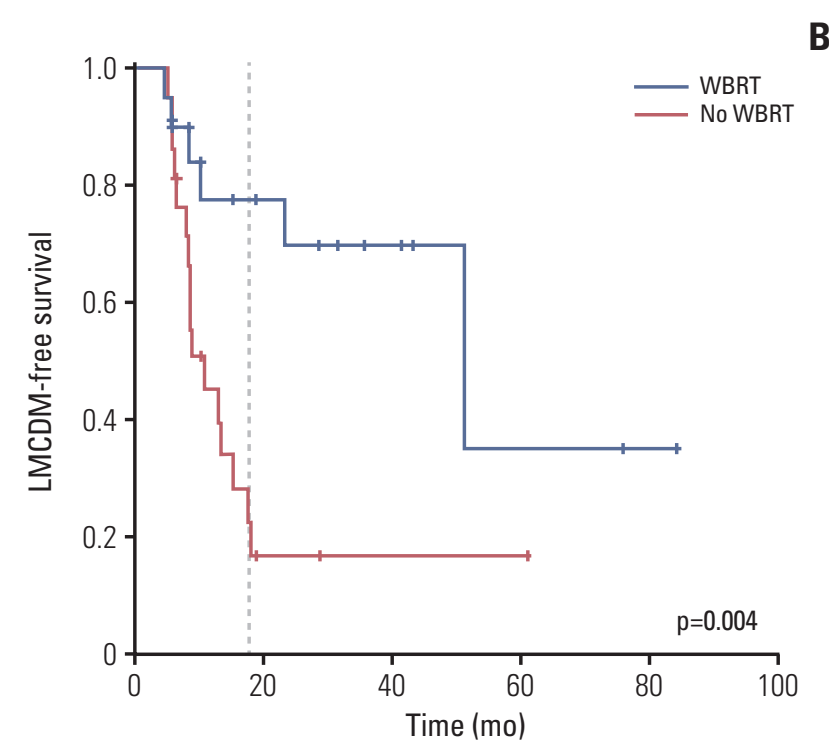

Fig. 3. Leptomeningeal carcinomatosis or dural metastasis-free survival (LMCDM-free survival) comparing three treatment groups (whole brain radiotherapy [WBRT] group [n=24], partial radiotherapy [PRT] group [n=10], and no radiotherapy [RT] group [n=17]) (A) and comparing two groups (WBRT [n=24] or not [n=27]) (B).

the WBRT group. LMCDM was less common in the WBRT group $(25 \%, 6 / 24$ patients) than in the no RT $(47 \%, 8 / 17$ patients) or PRT groups $(80 \%, 8 / 10$ patients). Patterns of failures according to the treatment groups and dose-fractionation of RT are listed in S2 Table.

\section{Leptomeningeal carcinomatosis or dural metastasis}

At a median follow-up of 12.4 months (range, 2.3 to 83.6 months), LMCDM was detected in 22 out of 51 patients (43\%) after surgery. The median time to the diagnosis of LMCDM after surgery was 8.6 months (range, 4.8 to 51.2 months). The 18-month LMCDM-free survival (LMCDM-FS) rate was higher in the WBRT group $(77.5 \%)$ than in the PRT group (30.0\%, $\mathrm{p}=0.021$ ) and no RT group (13.6\%, $\mathrm{p}=0.007)$ (Fig. 3A). There was no difference in 18-month LMCDM-FS between the PRT and no RT groups ( $\mathrm{p}=0.449)$. When patients were divided into two groups according to whether they received WBRT or not, the LMCDM-FS was significantly better in the WBRT group than in the no WBRT group (77.5\% vs. $22.6 \%$, $\mathrm{p}=0.004)$ (Fig. 3B).

Table 2 shows the results of univariate analyses aimed at identifying which factors were potentially associated with the incidence of LMCDM. Tumors adjacent to the CSF flow were associated with increased risk of LMCDM. Postoperative WBRT and systemic treatment decreased the incidence of LMCDM. Tumor size, extent of resection, and biological subtype of the primary tumor were not associated with
LMCDM upon univariate analyses. In the WBRT group, there were no differences in the incidence of LMCDM according to the dose-fractionation scheme of the WBRT. Multivariate analysis showed that WBRT and systemic treatment were significantly associated with reduced incidence of LMCDM. The HR relative to WBRT was 5.2 (95\% CI, 1.9 to $14.8 ; \mathrm{p}=0.009)$ for PRT and 2.7 (95\% CI, 0.9 to $7.9 ; \mathrm{p}=0.122)$ for no RT.

\section{LR and OS}

The 18-month LR-free survival was $83.6 \%, 75.0 \%$, and $14.1 \%$ in the WBRT, PRT, and no RT groups, respectively $(\mathrm{p}=0.002)$. The LR-free survival was significantly lower in the no RT group than in the other groups (Fig. 4).

The median OS in all patients was 28.7 months. The median OS was 24.0 months in the WBRT group and 37.7 months in the PRT group, while it was 11.6 months in the no RT group. However, the OS did not differ significantly among the three groups ( $p=0.255$ ) (Fig. 5). The OS values did not differ among biological subtypes of the primary tumor upon univariate analysis $(\mathrm{p}=0.562)$. The median OS was longer in patients with no or stable extracranial disease than those with progressive disease (median, 32.6 months vs. 11.6 months; $\mathrm{p}=0.067$ ), although the difference was not statistically significant. Systemic treatment was also associated with a longer median survival time (yes, 32.6 months vs. no, 24.0 months; $\mathrm{p}=0.019$ ). 
Table 2. Univariate and multivariate analysis for LMCDM-FS

\begin{tabular}{|c|c|c|c|c|c|c|}
\hline \multirow[b]{2}{*}{ Characteristic } & \multirow{2}{*}{$\begin{array}{c}\text { No. of } \\
\text { patients }\end{array}$} & \multicolumn{2}{|c|}{ Univariate analysis } & \multicolumn{3}{|c|}{ Multivariate analysis } \\
\hline & & $\begin{array}{c}\text { 18-Month } \\
\text { LMCDM-FS (\%) }\end{array}$ & p-value & $\begin{array}{c}\text { Hazard } \\
\text { ratio }^{\text {b) }}\end{array}$ & $95 \% \mathrm{CI}$ & p-value ${ }^{c}$ \\
\hline \multicolumn{7}{|l|}{ Extracranial disease status } \\
\hline None or stable & 35 & 42.1 & 0.113 & & & \\
\hline Progressive & 16 & 64.8 & & & & \\
\hline \multicolumn{7}{|c|}{ Biological subtype of primary tumor } \\
\hline ER + and / or PR+, HER2- & 7 & 53.6 & 0.882 & & & \\
\hline HER2-positive & 19 & 44.1 & & & & \\
\hline Triple negative & 13 & 58.3 & & & & \\
\hline \multicolumn{7}{|l|}{ No. of brain metastases } \\
\hline 1 & 36 & 45.1 & 0.191 & & & \\
\hline $2-3$ & 15 & 60.0 & & & & \\
\hline \multicolumn{7}{|l|}{ Size $(\mathrm{cm})$} \\
\hline$<3.5$ & 23 & 51.3 & 0.906 & & & \\
\hline$\geq 3.5$ & 27 & 41.1 & & & & \\
\hline \multicolumn{7}{|l|}{ Adjacent to CSF flow ${ }^{\text {d) }}$} \\
\hline No & 10 & 80.0 & 0.050 & 1.0 & - & - \\
\hline Yes & 40 & 40.3 & & 2.3 & $0.4-13.5$ & 0.456 \\
\hline \multicolumn{7}{|l|}{ Extent of resection } \\
\hline GTR & 40 & 42.6 & 0.120 & & & \\
\hline STR & 11 & 71.4 & & & & \\
\hline \multicolumn{7}{|c|}{$\begin{array}{l}\text { Systemic treatment after brain surgery } \\
\text { and before the development of LMCDM }\end{array}$} \\
\hline No & 21 & 8.0 & $<0.001$ & 1.0 & - & - \\
\hline Targeted $\mathrm{Tx} \pm \mathrm{CT} x$ & 13 & 69.8 & & 0.1 & $0.0-0.3$ & $<0.001$ \\
\hline CTx & 17 & 67.5 & & 0.1 & $0.0-0.3$ & $<0.001$ \\
\hline \multicolumn{7}{|l|}{ Postoperative RT } \\
\hline WBRT & 24 & 77.5 & 0.013 & 1.0 & - & - \\
\hline PRT & 10 & 30.0 & & 5.2 & $1.9-14.8$ & 0.009 \\
\hline No RT & 17 & 13.6 & & 2.7 & $0.9-7.9$ & 0.122 \\
\hline \multicolumn{7}{|l|}{ Postoperative WBRT } \\
\hline Yes & 24 & 77.5 & 0.004 & & & \\
\hline No & 27 & 22.6 & & & & \\
\hline \multicolumn{7}{|l|}{ WBRT dose-fractionation } \\
\hline $25 \mathrm{~Gy} / 10 \mathrm{fx}$ & 9 & 65.6 & 0.945 & & & \\
\hline $30 \mathrm{~Gy} / 12 \mathrm{fx}$ & 6 & 100.0 & & & & \\
\hline $30 \mathrm{~Gy} / 10 \mathrm{fx}$ & 9 & 71.4 & & & & \\
\hline
\end{tabular}

LMCDM-FS, leptomeningeal carcinomatosis or dural metastasis-free survival; CI, confidence interval; ER, estrogen receptor; PR, progesterone receptor; HER2, human epidermal growth factor receptor 2; CSF, cerebrospinal fluid; GTR, gross total resection; STR, subtotal resection; Tx, therapy; CTx, chemotherapy; RT, radiotherapy; WBRT, whole brain radiotherapy; PRT, partial radiotherapy; $\mathrm{fx}$, fractions. ${ }^{\mathrm{a})} \mathrm{Log}$-rank test, ${ }^{\mathrm{b})}$ Hazard ratio refers to the risk of leptomeningeal carcinomatosis or dural metastasis per unit time, ${ }^{\mathrm{c}}$ Cox proportional hazard model (backward likelihood ratio), ${ }^{\mathrm{d}} \mathrm{n}=50$ (WBRT, 23; PRT, 10; no RT, 17).

\section{Subgroup analysis according to HER2 status}

In the 31 patients with HER2-positive primary tumors, systemic treatment including targeted therapy was associated with improved LMDCM-FS when compared with no sys- temic treatment $(\mathrm{p}=0.002)$. In the 20 patients with HER2-negative primary tumor, systemic treatment decreased the incidence of LMCDM ( $<$ 0.001) (S3 Fig.).

Systemic treatment including targeted therapy was also associated with better OS than no systemic treatment in the 


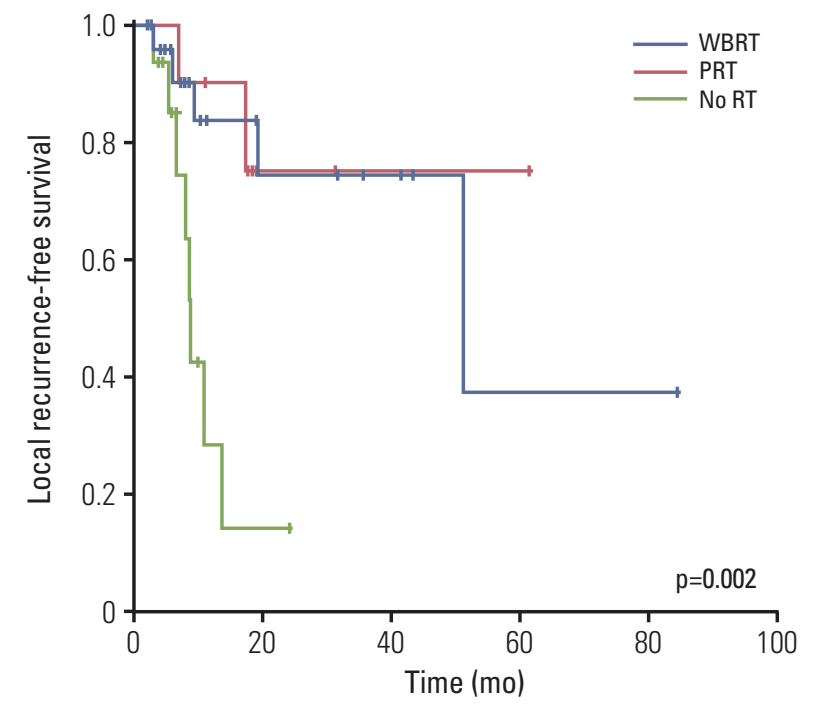

Fig. 4. Local recurrence-free survival for whole brain radiotherapy (WBRT) group $(\mathrm{n}=24)$, partial radiotherapy (PRT) group $(\mathrm{n}=10)$, and no radiotherapy (RT) group $(\mathrm{n}=17)$.

HER2-positive subgroup of patients $(\mathrm{p}=0.047)$. In the HER2negative subgroup of patients, systemic treatment did not improve median OS ( $\mathrm{p}=0.159$ ) (S4 Fig.).

\section{Discussion}

This study was conducted to assess the effects of postoperative WBRT on LMCDM and to identify the risk factors associated with LMCDM in patients who underwent surgical resection of brain metastases from breast cancer. LMCDM occurred in 22/51 patients ( $43 \%$ ) and was the most common pattern of disease recurrence after surgical resection in this study. Patients who received WBRT had less LMCDM than those who received PRT. These results are consistent with those of previous retrospective studies that compared the outcomes of WBRT and PRT in postoperative settings. Patel et al. [8] reported 18-month LMC rates of $13 \%$ with WBRT and $31 \%$ with stereotactic radiosurgery (SRS) ( $p=0.045$ ). Hsieh et al. [9] also reported a lower rate of LMC with WBRT $(\mathrm{p}=0.02)$.

Our study also demonstrated an association between the presence of a tumor adjacent to CSF flow and increased risk of LMCDM. Several other studies found an association between tumor location and LMC. Some studies have reported that the rate of LMC after surgical resection was

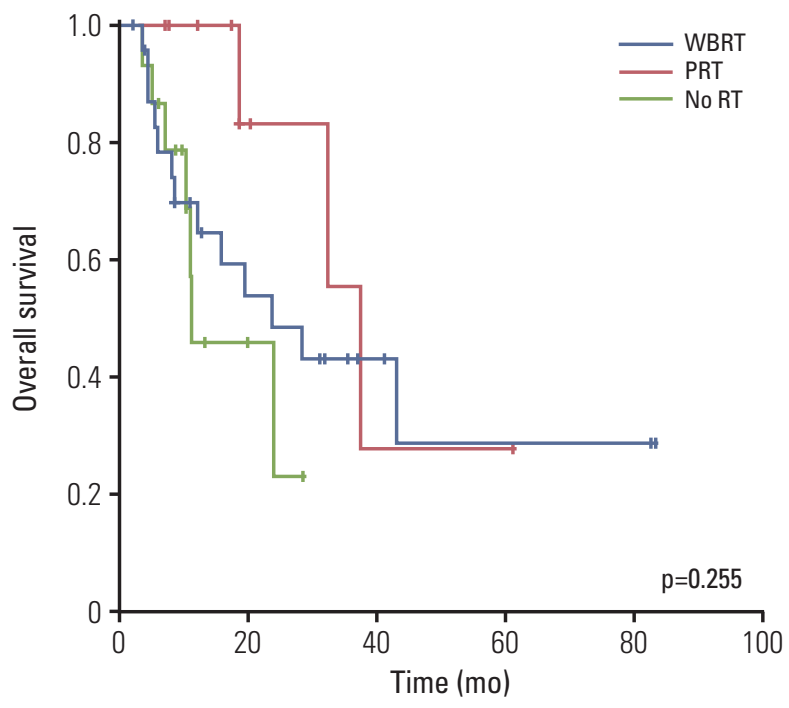

Fig. 5. Overall survival for whole brain radiotherapy (WBRT) group ( $\mathrm{n}=24)$, partial radiotherapy (PRT) group $(\mathrm{n}=10)$, and no radiotherapy (RT) group $(\mathrm{n}=17)$.

higher for posterior fossa tumors than for supratentorial tumor $[13,14]$. Theoretically, there is a greater chance of CSF exposure during resection of posterior fossa tumors. Ahn et al. [12] classified tumor location relative to CSF flow. Their study showed an increased risk of LMCDM in patients with a tumor adjacent to the CSF flow (HR, 9.00; $p<0.01$ ), consistent with our results. Although this association was not significant in our multivariate analysis, we assume that the patients with tumors adjacent to CSF flow could be at a high risk of LMCDM and that postoperative WBRT needs to be considered.

Concern about the risk of neurotoxicity from WBRT is the main reason postoperative local RT is often applied instead of WBRT in many clinical situations $[6,7,15,16]$. However, intracranial disease progression is another important cause of neurocognitive deterioration [17]. Occurrence of LMCDM is associated with significant morbidity, and intensive treatment of LMCDM can markedly compromise patient quality of life. Thus, it is particularly important to identify patients at higher risk of LMCDM and prevent the development of LMCDM, especially among those in whom long-term survival is expected. In the WBRT group, various dose-fractionation schemes ( $30 \mathrm{~Gy} / 10 \mathrm{fx}, 30 \mathrm{~Gy} / 12 \mathrm{fx}$, or $25 \mathrm{~Gy} / 10 \mathrm{fx}$ ) were used, all of which resulted in favorable outcomes. In patients with small cell lung cancer, prophylactic cranial irradiation of $25 \mathrm{~Gy}$ in $10 \mathrm{fx}$ has been a standard regimen because increased risk of chronic neurotoxicity at higher doses had been reported [18]. Taken together, these results 
indicate that postoperative WBRT 25 Gy in $10 \mathrm{fx}$ followed by local boost can be considered after surgical resection of brain metastases to minimize neurotoxicity while achieving an acceptable tumor control.

The incidence of LMCDM was higher in our study than the incidence of LMC in previous reports, which included patients with brain metastases from any primary tumor $[19,20]$. The broader criterion for the diagnosis of LMCDM used in our study may explain the higher incidence. Twenty two patients developed LMCDM, eight of whom were diagnosed with dural metastasis. Like the patients with LMC, those with dural metastasis eventually require salvage therapy, including WBRT and intrathecal chemotherapy, and are at greater risk of toxicity because of these aggressive treatments. Thus, multiple enhancing dural nodules were also analyzed as LMCDM rather than DBR. Because the incidence of both LMC and dural metastasis can be affected by the extent of postoperative RT, we expected this broad criterion of LMCDM would be useful to identify patients in whom WBRT needs to be considered.

The lower incidence of LMCDM in the WBRT group was not associated with prolonged survival. The median survival was longer in PRT groups (37.7 months) than those in the no RT (11.6 months) or WBRT group (24.0 months), but this difference was not statistically significant. We assume that these results were caused by differences in baseline characteristics among groups. In the PRT group, there were more patients with a Breast-GPA score of 3.5-4.0, who were expected to have better prognosis than others, although this difference was not statistically significant. There was also a possibility that physicians had selected patients with good prognostic factors including Breast-GPA and were expected to live longer to receive PRT rather than WBRT to avoid neurotoxicity. The longer survival time in the PRT group suggests that patients might have had a greater chance to develop LMCDM because they lived longer than others. This possibility cannot be thoroughly investigated based on the present study. However, considering that the median time to develop LMCDM was only 8.6 months, the median survival time of the no RT or WBRT group was not too short to underestimate the LMCDM.

In addition to WBRT and tumor location, several other factors were reportedly associated with the development of LMC in previous studies. For example, primary tumor histology, size of resected lesion, piecemeal resection, and previous intracranial recurrence were associated with the incidence of LMC following surgical resection of brain metastases [12,20-24]. The number of brain metastasis and location of systemic disease progression were prognostic factors for LMC in patients who underwent upfront SRS for brain metastases $[19,25,26]$. In the current study, we found that systemic treatment after resection of brain metastases was sig- nificantly associated with reduced risk of LMCDM, with a HR of 0.1 compared to the patients who did not receive systemic treatment $(\mathrm{p}<0.001)$. These findings are contrary to the widely accepted concept that systemic treatment is less effective in patients with brain metastasis because of the blood-brain barrier (BBB).

However, there is evidence that some targeted agents can cross the BBB [27]. Lapatinib is known to cross the BBB because of its very low molecular weight $(581 \mathrm{Da})$, and several studies evaluating the effects of lapatinib in patients with metastatic brain tumor from the breast are ongoing (NCT01622868 and NCT01218529). This can explain the improved LMCDM-FS in patients with HER2-positive primary tumor who underwent targeted therapies in this study. Among 12 patients who received targeted therapy, 11 were treated with lapatinib.

Patients with HER2-negative primary tumor also had an improved LMCDM-FS with chemotherapeutic agents upon subgroup analysis. Because all patients in this study underwent surgical resection of brain metastasis, the systemic agents might be able to cross the disrupted BBB, and hence eliminate the microscopically seeded tumor cells in the CSF. However, the heterogeneity of the regimens and diverse clinical situations make it difficult to determine the impact of systemic treatment on the development of LMCDM in the current study.

The strength of our study lies in the homogeneous study population. All of the patients underwent surgical resection of brain metastases from breast cancer. It should be noted that most of the previous studies included mixed populations of patients with metastases from a variety of primary solid tumor types, and more than half of the study populations in prior studies had primary lung cancer. To date, very few studies have determined the incidence of LMC after resection of brain metastases in breast cancer patients. Brain metastases of breast cancer should be separated from those of other primary tumors because of the much longer survival time. De Ieso et al. [28] reported that intensive treatment, including surgery and / or stereotactic RT, of brain metastases can result in long-term survival of more than 2 years in breast cancer patients with a limited number of brain metastases. Thus, both the effective disease control and preservation of quality of life should be pursued in these patients, and determining the proper extent of RT after surgical resection of brain metastasis is of particularly great importance.

There are limitations inherent to retrospective studies. The small number of patients and the baseline imbalance in patient characteristics among treatment groups might have biased the apparent effects of the WBRT. The effects of several factors known to increase the risk of LMC in previous studies, including the location and size of the tumor or the number of lesions, were not fully demonstrated in this study. 
Given the inherent bias in retrospective analyses, a large prospective study of breast cancer patients with brain metastases is needed to precisely define high-risk patients who need to receive WBRT after surgery.

\section{Conclusion}

WBRT improved the LMCDM-FS rate after resection of brain metastases compared to PRT in breast cancer patients. Patients with tumors adjacent to the CSF flow are at higher risk of LMCDM, and these patients might need to be treated with postoperative WBRT. The effects of systemic treatment on LMCDM needs to be evaluated in further studies.

\section{Electronic Supplementary Material}

Supplementary materials are available at Cancer Research and Treatment website (http:// www.e-crt.org).

\section{Conflicts of Interest}

Conflict of interest relevant to this article was not reported.

\section{Author Details}

${ }^{1}$ Proton Therapy Center, Research Institute and Hospital, National Cancer Center, Goyang, ${ }^{2}$ Department of Radiation Oncology, Yonsei University College of Medicine, Seoul, ${ }^{3}$ Neuro-Oncology Clinic, Research Institute and Hospital, National Cancer Center, Goyang, ${ }^{4}$ Department of Neurosurgery, Yonsei University College of Medicine, Seoul, ${ }^{5}$ Department of Neurology, Research Institute and Hospital, National Cancer Center, Goyang, ${ }^{6}$ Center for Breast Cancer, Research Institute and Hospital, National Cancer Center, Goyang, Korea

\section{References}

1. Barnholtz-Sloan JS, Sloan AE, Davis FG, Vigneau FD, Lai P, Sawaya RE. Incidence proportions of brain metastases in patients diagnosed (1973 to 2001) in the Metropolitan Detroit Cancer Surveillance System. J Clin Oncol. 2004;22:2865-72.

2. Noordijk EM, Vecht CJ, Haaxma-Reiche H, Padberg GW, Voormolen JH, Hoekstra FH, et al. The choice of treatment of single brain metastasis should be based on extracranial tumor activity and age. Int J Radiat Oncol Biol Phys. 1994;29:711-7.

3. Patchell RA, Tibbs PA, Walsh JW, Dempsey RJ, Maruyama Y, Kryscio RJ, et al. A randomized trial of surgery in the treatment of single metastases to the brain. N Engl J Med. 1990;322: 494-500.

4. Kocher M, Soffietti R, Abacioglu U, Villa S, Fauchon F, Baumert BG, et al. Adjuvant whole-brain radiotherapy versus observation after radiosurgery or surgical resection of one to three cerebral metastases: results of the EORTC 22952-26001 study. J Clin Oncol. 2011;29:134-41.

5. Patchell RA, Tibbs PA, Regine WF, Dempsey RJ, Mohiuddin M, Kryscio RJ, et al. Postoperative radiotherapy in the treatment of single metastases to the brain: a randomized trial. JAMA. 1998;280:1485-9.

6. Choi CY, Chang SD, Gibbs IC, Adler JR, Harsh GR 4th, Lieberson $\mathrm{RE}$, et al. Stereotactic radiosurgery of the postoperative resection cavity for brain metastases: prospective evaluation of target margin on tumor control. Int J Radiat Oncol Biol Phys. 2012;84:336-42.

7. Robbins JR, Ryu S, Kalkanis S, Cogan C, Rock J, Movsas B, et al. Radiosurgery to the surgical cavity as adjuvant therapy for resected brain metastasis. Neurosurgery. 2012;71:937-43.

8. Patel KR, Prabhu RS, Kandula S, Oliver DE, Kim S, Hadjipanayis $\mathrm{C}$, et al. Intracranial control and radiographic changes with adjuvant radiation therapy for resected brain metastases: whole brain radiotherapy versus stereotactic radiosurgery alone. J Neurooncol. 2014;120:657-63.

9. Hsieh J, Elson P, Otvos B, Rose J, Loftus C, Rahmathulla G, et al. Tumor progression in patients receiving adjuvant wholebrain radiotherapy vs localized radiotherapy after surgical resection of brain metastases. Neurosurgery. 2015;76:411-20.

10. Sperduto PW, Kased N, Roberge D, Xu Z, Shanley R, Luo X, et al. Effect of tumor subtype on survival and the graded prognostic assessment for patients with breast cancer and brain metastases. Int J Radiat Oncol Biol Phys. 2012;82:2111-7.

11. Dawood S, Gonzalez-Angulo AM, Albarracin C, Yu TK, Hortobagyi GN, Buchholz TA, et al. Prognostic factors of survival in the trastuzumab era among women with breast cancer and brain metastases who receive whole brain radiotherapy: a sin- 
gle-institution review. Cancer. 2010;116:3084-92.

12. Ahn JH, Lee SH, Kim S, Joo J, Yoo H, Lee SH, et al. Risk for leptomeningeal seeding after resection for brain metastases: implication of tumor location with mode of resection. J Neurosurg. 2012;116:984-93.

13. Dosoretz DE, Blitzer PH, Russell AH, Wang CC. Management of solitary metastasis to the brain: the role of elective brain irradiation following complete surgical resection. Int J Radiat Oncol Biol Phys. 1980;6:1727-30.

14. Norris LK, Grossman SA, Olivi A. Neoplastic meningitis following surgical resection of isolated cerebellar metastasis: a potentially preventable complication. J Neurooncol. 1997;32: 215-23.

15. Brennan C, Yang TJ, Hilden P, Zhang Z, Chan K, Yamada Y, et al. A phase 2 trial of stereotactic radiosurgery boost after surgical resection for brain metastases. Int J Radiat Oncol Biol Phys. 2014;88:130-6.

16. Chang EL, Wefel JS, Hess KR, Allen PK, Lang FF, Kornguth DG, et al. Neurocognition in patients with brain metastases treated with radiosurgery or radiosurgery plus whole-brain irradiation: a randomised controlled trial. Lancet Oncol. 2009; 10:1037-44.

17. Regine WF, Huhn JL, Patchell RA, St Clair WH, Strottmann J, Meigooni A, et al. Risk of symptomatic brain tumor recurrence and neurologic deficit after radiosurgery alone in patients with newly diagnosed brain metastases: results and implications. Int J Radiat Oncol Biol Phys. 2002;52:333-8.

18. Wolfson AH, Bae K, Komaki R, Meyers C, Movsas B, Le Pechoux $C$, et al. Primary analysis of a phase II randomized trial Radiation Therapy Oncology Group (RTOG) 0212: impact of different total doses and schedules of prophylactic cranial irradiation on chronic neurotoxicity and quality of life for patients with limited-disease small-cell lung cancer. Int J Radiat Oncol Biol Phys. 2011;81:77-84.

19. Huang AJ, Huang KE, Page BR, Ayala-Peacock DN, Lucas JT Jr, Lesser GJ, et al. Risk factors for leptomeningeal carcinomatosis in patients with brain metastases who have previously undergone stereotactic radiosurgery. J Neurooncol. 2014;120: 163-9.

20. Ojerholm E, Lee JY, Thawani JP, Miller D, O'Rourke DM, Dorsey JF, et al. Stereotactic radiosurgery to the resection bed for intracranial metastases and risk of leptomeningeal carcinomatosis. J Neurosurg. 2014;121 Suppl:75-83.

21. Atalar B, Modlin LA, Choi CY, Adler JR, Gibbs IC, Chang SD, et al. Risk of leptomeningeal disease in patients treated with stereotactic radiosurgery targeting the postoperative resection cavity for brain metastases. Int J Radiat Oncol Biol Phys. 2013;87:713-8

22. Jung JM, Kim S, Joo J, Shin KH, Gwak HS, Lee SH. Incidence and risk factors for leptomeningeal carcinomatosis in breast cancer patients with parenchymal brain metastases. J Korean Neurosurg Soc. 2012;52:193-9.

23. Suki D, Abouassi H, Patel AJ, Sawaya R, Weinberg JS, Groves MD. Comparative risk of leptomeningeal disease after resection or stereotactic radiosurgery for solid tumor metastasis to the posterior fossa. J Neurosurg. 2008;108:248-57.

24. Suki D, Hatiboglu MA, Patel AJ, Weinberg JS, Groves MD, Mahajan A, et al. Comparative risk of leptomeningeal dissemination of cancer after surgery or stereotactic radiosurgery for a single supratentorial solid tumor metastasis. Neurosurgery. 2009;64:664-74.

25. Jo KI, Lim DH, Kim ST, Im YS, Kong DS, Seol HJ, et al. Leptomeningeal seeding in patients with brain metastases treated by gamma knife radiosurgery. J Neurooncol. 2012;109:293-9.

26. Trifiletti DM, Romano KD, Xu Z, Reardon KA, Sheehan J. Leptomeningeal disease following stereotactic radiosurgery for brain metastases from breast cancer. J Neurooncol. 2015;124: 421-7.

27. Nelson MH, Dolder CR. Lapatinib: a novel dual tyrosine kinase inhibitor with activity in solid tumors. Ann Pharmacother. 2006;40:261-9.

28. De Ieso PB, Schick U, Rosenfelder N, Mohammed K, Ross GM. Breast cancer brain metastases: a 12 year review of treatment outcomes. Breast. 2015;24:426-33. 